\title{
Stimulation hormonale, in vivo, de l'ovaire d'anguille européenne au stade jaune
}

\author{
E Lopez *, YA Fontaine \\ avec l'assistance technique de F Lallier, N Le Belle, B Vidal \\ URA CNRS 90, laboratoire de physiologie générale et comparée du Muséum national \\ d'Histoire naturelle, 7, rue Cuvier, 75231 Paris, Cedex 05, France
}

(Reçu le $1^{\text {er }}$ mars 1990; accepté le 15 juin 1990)

\begin{abstract}
Résumé - L'ovaire, immature, d'anguilles au stade jaune est stimulé à la suite d'un traitement in vivo de longue durée par un extrait d'hypophyse de carpe riche en gonadotropine de type II. Les follicules ovariens d'anguilles jaunes traitées montrent une accumulation de vacuoles lipidiques dans le cytoplasme ovocytaire et une limitante folliculaire épaisse (ils ressemblent par ces caractères à ceux d'anguilles argentées étudiées précédemment). Le traitement induit aussi une augmentation significative mais limitée du rapport gonadosomatique et de la taille moyenne des follicules (les valeurs atteintes sont bien inférieures à celles mesurées précédemment chez des anguilles argentées). Aucun changement de la livrée n'a été observé. Dans nos conditions expérimentales, une stimulation gonadotrope de l'anguille jaune entraîne donc certaines des modifications ovariennes intervenant physiologiquement au cours de l'argenture, mais non l'ensemble de ces modifications.
\end{abstract}

hypophyse / gonadotropine / ovaire / poisson / anguille jaune

Summary - Hormonal in vivo stimulation of the ovary in the yellow European eel. The immature ovary of yellow eel was stimulated by a long-term treatment with a gonadotropin II rich extract of carp pituitary. Ovarian follicles of treated yellow eels showed an accumulation of lipidic vacuoles in the oocyte cytoplasm and a thickened follicular envelope (in this regard, they resembled the ovarian follicles of previously studied silver eels). The treatment also resulted in a significant but limited increase in the gonadosomatic ratio and mean follicle size (these values were much lower than those measured in silver eels). No change in pigmentation or general morphology was observed. Thus, under our experimental conditions, a gonadotropic stimulation of the yellow eel leads to some but not the totality of the ovarian modifications which occur physiologically during silvering.

pituitary / gonadotropin / ovary / fish / yellow eel

\section{INTRODUCTION}

Le cycle vital si particulier de l'anguille européenne (Anguilla anguilla) inclut une longue phase de croissance en eau douce, durant laquelle les poissons présentent une livrée jaune caractéristique. Chaque année à la fin de l'été et à des âges très variables (5-20 ans), des anguilles jaunes se transforment en anguilles argentées qui vont entreprendre la migration marine de reproduction (Bertin, 1951; Tesch, 1979, Fontaine, 1989).

Cette "argenture" s'accompagne de nombreuses modifications anatomophysiologiques (Fontaine, 1989).

\footnotetext{
* Correspondance et tirés à part.
} 
Certains aspects de la structure de l'ovaire de l'anguille jaune ont été décrits dans quelques articles consacrés plus spécialement à la différenciation sexuelle (Rodolico, 1933; d'Ancona, 1943; Colombo et al, 1984); les données indiquent un développement progressif de l'ovaire, des petites anguilles jaunes aux anguilles argentées. Chez ces dernières, l'ovaire est en début de vitellogenèse (Fontaine et al, 1976; Le Menn, Burzawa-Gérard, 1985) et donc encore très loin de la maturité sexuelle; de plus il est bloqué dans cet état, par suite d'un manque de sécrétion de gonadotropine hypophysaire (Dufour et al, 1988), et il le reste si la migration est empêchée.

Les phénomènes intervenant pendant la période d'argenture, et leur déterminisme, sont encore mal connus. Dans cette note, nous décrirons la morphologie de l'ovaire d'anguilles jaunes dont le poids et les conditions de pêche étaient similaires à ceux d'anguilles argentées. De plus, nous montrerons que ces ovaires sont sensibles à une stimulation hormonale in vivo par un extrait hypophysaire riche en gonadotropine et nous comparerons les résultats de cette stimulation aux changements observés lors de l'argenture naturelle.

\section{MATÉRIEL ET MÉTHODES}

Les anguilles sont pêchées au début de l'automne, dans un étang de Péronne (Somme). Les individus choisis pour leur livrée jaune caractéristique sont gardés au laboratoire en eau courante, à jeûn, et sacrifiés dans les 2 mois qui suivent la pêche. Un lot est au préalable traité par un extrait salin $(\mathrm{NaCl} 0,10 \mathrm{~mol} / \mathrm{l})$ d'hypophyse de carpe (EHC), 3 fois par semaine pendant 7 semaines. Chaque injection, intrapéritonéale $(0,5 \mathrm{ml})$, correspond à 2,5 mg d'hypophyse de carpe, équivalent à environ $160 \mu \mathrm{g}$ de gonadotropine (GHT) de type II (Fontaine et al, 1976). Après sacrifice et pesée des animaux, les ovaires sont pesés et le rapport gonadoso- matique $R G S=$ [poids de l'ovaire $/$ poids $\mathrm{du}$ corps $x$ 100)] est calculé. Les échantillons d'ovaire prélevés dans la région médiane sont fixés dans le Bouin aqueux et traités par les méthodes histologiques classiques. Les coupes sont colorées par la coloration trichrome de Cleveland Wolfe. Les mesures du diamètre folliculaire moyen sont effectuées, avec un micromètre oculaire, sur cinquante follicules pris au hasard.

\section{RÉSULTATS}

Aucun changement de livrée n'a été observé chez les animaux traités.

Le tableau I résume les données obtenues pour le $R G S$ et le diamètre folliculaire $\left(D_{\mathrm{F}}\right)$. Le traitement par l'EHC entraîne une augmentation significative de ces 2 paramètres. Le volume folliculaire moyen $\left(V_{F}\right)$, calculé à partir du $D_{\mathrm{F}}$ moyen en supposant les follicules sphériques est (en $10^{-3} \mathrm{~mm}^{3}$ ) 0,119 et 0,463 respectivement pour témoins et traités. Ainsi sous l'influence du traitement hypophysaire, le volume folliculaire a plus augmenté (+ $289 \%$ ) que le RGS (+ 84\%).

Tableau I. Effets d'un traitement chronique par un extrait d'hypophyse de carpe sur l'ovaire d'anguille jaune.

Témoins

Traitées *

(6)

(4)

$\begin{array}{lcr}\text { Poids du corps (g) } & 202 \pm 17 & 247 \pm 38 \text { (a) } \\ \text { RGS (\%) } & 0,38 \pm 0,03 & 0,70 \pm 0,05^{\text {(b) }} \\ D_{F}(\mu \mathrm{m}) & 61,0 \pm 5,0 & 96,0 \pm 3,2^{\text {(c) }}\end{array}$

- 2,5 mg d'extrait d'hypophyse de carpe, 3 fois par semaine durant 7 semaines.

(a) $P>0,05$ (test de Mann-Whitney); (b) $P<0,05$ (test de Mann-Whitney après transformation Arcsin- $\downarrow$ ); (c) $P$ $<0,05$ (test de Mann-Whitney). 
Chez les anguilles témoins, les follicules sont groupés en cordons, ils sont limités par une fine thèque conjonctive peu distincte de la membrane ovocytaire (fig 1). Le cytoplasme des ovocytes est granuleux et très dense, il contient des inclusions basophiles (figs $3,4,5$ ) correspondant sans doute aux corps sidérophiles décrits par Rodolico (1933) et Colombo et al (1984). Le noyau a de gros nucléoles répartis sans ordre défini (fig 1, 3, 4). Le tissu conjonctif délimitant les cordons folliculaires s'insinue entre les follicules (fig 1); on distingue ainsi dans les espaces interfolliculaires un tissu interstitiel lâche constitué de fibrocytes avec des capillaires sanguins (fig 4). Chez les anguilles traitées, la structure en cordons reste inchangée; en revanche, le cytoplasme ovocytaire est très modifié. On note l'apparition de nombreuses vacuoles lipidiques qui envahissent l'ensemble du cytoplasme dont la périphérie devient granuleuse et plus dense (figs 2,6 ); les corps sidérophiles disparaissent. On n'observe pas de granulosa distincte, toutefois, la limitante folliculaire (thèque + membrane ovocytaire) est très épaissie et on distingue quelques cellules thécales.

\section{DISCUSSION ET CONCLUSIONS}

Les ovaires des anguilles jaunes témoins sont caractérisés par l'absence de vitellogenèse, ce qui correspond au stade 2 défini chez d'autres poissons (Yamazaki, 1965; Le Menn, Burzawa-Gérard, 1985). lls se distinguent nettement de ceux d'anguilles argentées comme le montre la comparaison avec les données précédemment obtenues chez des poissons (de même origine) à ce stade (Fontaine et al, 1976). Cette comparaison permet d'évaluer les changements intervenant durant l'argenture. II s'agit d'une augmentation du RGS et du diamètre folliculaire (qui atteignent respectivement $1,86 \pm 0,13$ et $317 \pm 2 \mu \mathrm{m}$ ) ainsi que de transformations importantes de l'aspect des follicules.

Nous avons montré ici que l'ovaire des anguilles jaunes est clairement stimulé in vivo par un traitement chronique à l'EHC. Par certains aspects, les changements observés sont voisins de ceux intervenant durant l'argenture : disparition des corps sidérophiles, apparition des nombreuses vésicules lipidique, épaississement des limitantes folliculaires. Le cytoplasme périphérique apparaît même plus dense et granuleux chez les anguilles jaunes traitées que chez les anguilles argentées témoins. La stimulation est certainement due, au moins en partie, à la GTH II, abondante dans l'extrait hypophysaire utilisé; en effet, cette hormone est capable d'augmenter considérablement, in vitro, le monophosphate cyclique d'adénosine dans des fragments d'ovaire d'anguille jaune (Fontaine-Bertrand et al, 1978).

Cependant, le RGS et le $\mathrm{D}_{\mathrm{F}}$ sont loin, chez les anguilles jaunes traitées, d'atteindre des valeurs mesurées chez les anguilles argentées (cf supra).

De même, chez des anguilles jaunes traitées par l'EHC pendant seulement 3 semaines, Leloup-Hatey et al (1989) n'ont observé aucune modification du RGS tandis que l'estradiol plasmatique était très significativement élevé.

Ces discordances doivent résulter de l'absence, dans l'EHC utilisé, de quantités suffisantes d'hormones différentes de la GTH II mais intervenant aussi dans la stimulation de la croissance des follicules. Ces hormones, mises physiologiquement en jeu durant l'argenture, pourraient être la GTH I, qui est vraisemblablement peu abondante en période de maturation sexuelle chez les carpes adultes dont provient la poudre acétonique hypophysaire (cfles résultats obtenus chez des Salmoni- 

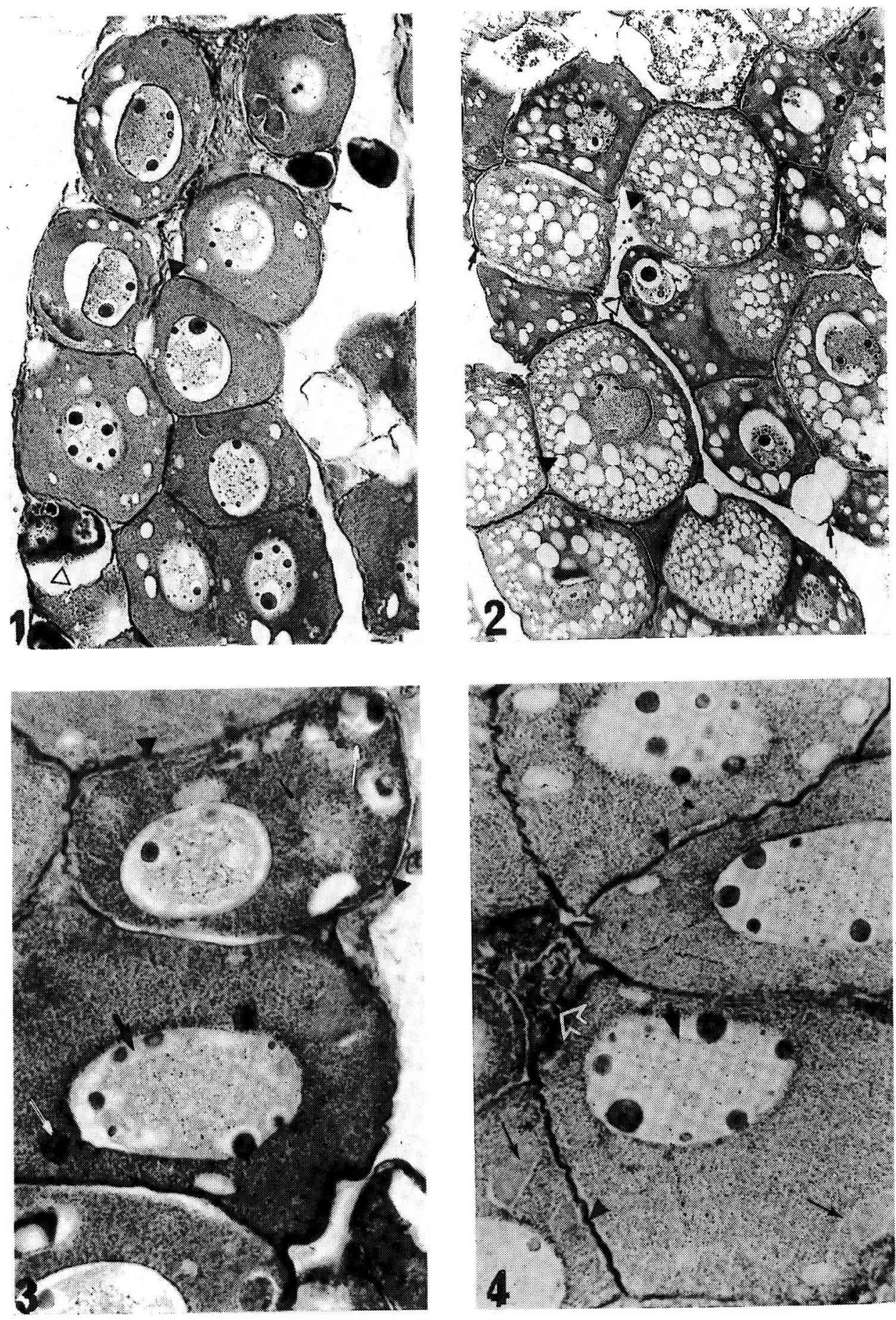

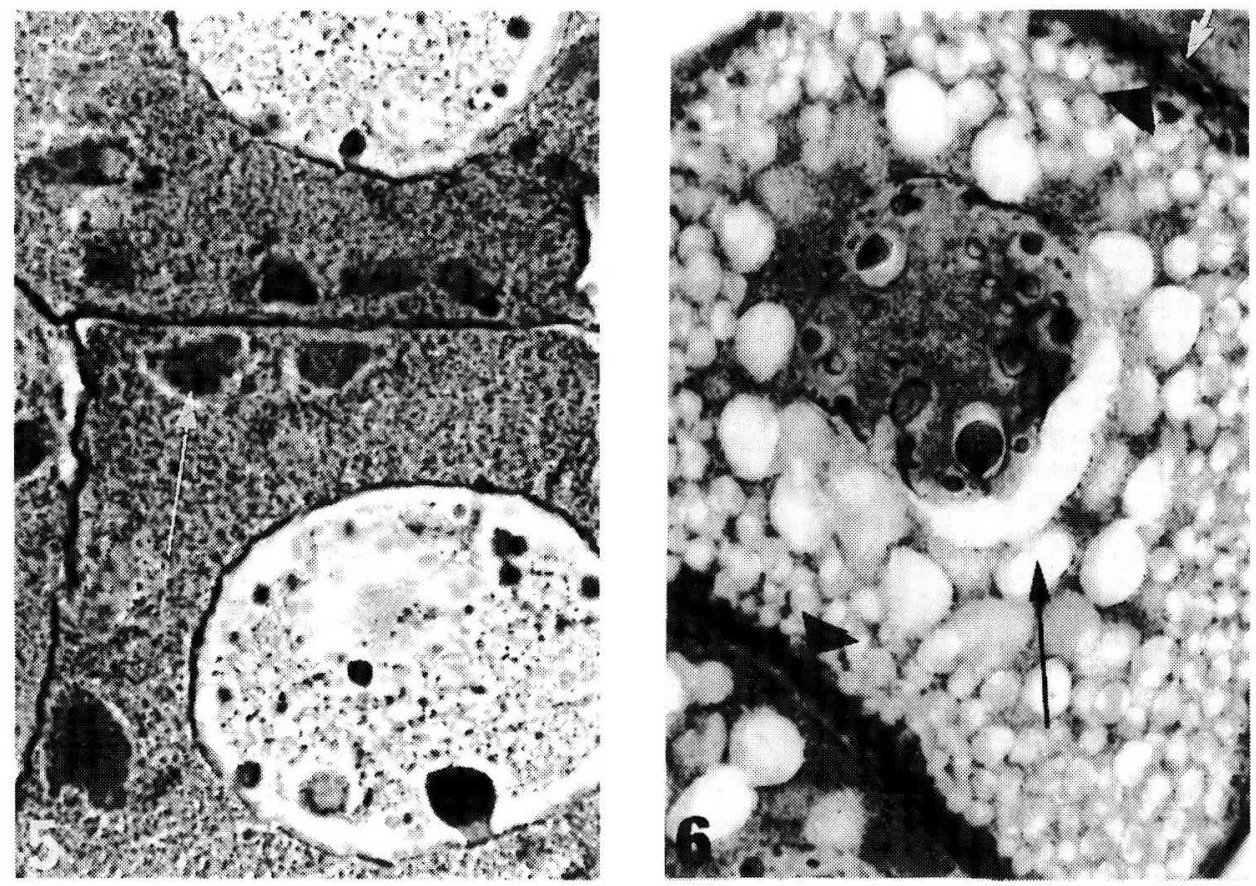

Figs 1-6. Coll Cleveland Wolfe.

Fig 1. Partie d'ovaire d'une anguille jaune témoin. Follicules groupés en cordons $(\rightarrow)$ séparés par des travées conjonctives $(-)$. Follicule atrétique $(\rightarrow)(x 100)$.

Flg 2. Partie d'ovaire d'une anguille jaune traitée. Follicules groupés en cordons $(\rightarrow)$. Follicules atrétiques $(\triangleright)$. Cytoplasmes ovocytaires très vacuolisés $(-)(x 100)$.

Figs 3-4. Follicules ovocytaires d'anguilles jaunes témoins. Limitante ovocytaire fine sans cellules distinctes $(\rightarrow$. Cytoplasme dense avec corps sidérophiles $\rightarrow$. Noyau et nucléoles $(\rightarrow)$. Tissu conjonctif interstitiel avec hématies $(\hookrightarrow)(\times 252)$.

Flgs 5-6. Follicule ovocytaire d'une anguille jaune témoin (5) comparé à celui d'une anguille traitée (6) $(\times 650)$. 5. Corps sidérophiles $(\rightarrow)$ dans cytoplasme dense. 6. Cytoplasme vacuolaire $(\rightarrow)$. Limitante folliculaire avec cellules thécales $(-)$. Cytoplasme périphérique granulaire dense $(\rightarrow)$.

dés par Suzuki et al, 1988) ou l'hormone de croissance. En effet, cette dernière (de Luze, Leloup, 1984) stimule la désiodation de thyroxine en triiodothyronine, ellemême sans doute impliquée dans l'argenture (Fontaine, 1975; Leloup et al, 1976).

Aucune modification de la livrée ni de la morphologie générale n'a été observée chez les anguilles jaunes traitées, ce qui suggère que la GTH II ne joue pas, de ces points de vue, un rôle essentiel. De nombreuses hormones, dont les hormones interrénaliennes (Epstein et al, 1971) jouent certainement un rôle dans le déterminisme du phénomène complexe de l'argenture (Fontaine, 1975). 


\section{RÉFÉRENCES}

d'Ancona U (1943) Nuove ricence sulla determinazione sessuale dell' anguilla. Arch Oceanogr Limnol 3, 159-269

Bertin L (1951) Les Anguilles. Payot, Paris

Colombo G, Grandi G, Rossi R (1984) Gonad differentiation and body growth in Anguilla anguilla L. J Fish Biol 24, 215-228

Dufour S, Lopez E, Le Belle N, Baloche S, Fontaine YA (1988) Stimulation of gonadotropin release and of ovarian development by the administration of a gonadoliberin agonist and of dopamine antagonists in female silver eel pretreated with estradiol. Gen Comp Endocrinol 70, 20-30

Epstein FH, Cynamon M, Mc Kay W (1971) Endocrine control of Na-K-ATPase and sea water adaptation in Anguilla rostrata. Gen Comp Endocrinol 16, 323-328

Fontaine M (1975) Physiological mechanisms in the migration of marine and amphihaline fish. Adv Mar Biol 13, 241-355

Fontaine YA (1989) Les anguilles : reproduction et migration. Oceanis 15, 197-206

Fontaine YA, Lopez E, Delerue-Le Belle N, Fontaine-Bertrand E, Lallier F, Salmon C (1976) Stimulation gonadotrope de l'ovaire chez l'anguille (Anguilla anguilla) hypophysectomisée. J Physiol, Paris 72, 871-892

Fontaine-Bertrand E, Salmon C, Fontaine YA, Delerue-Le Belle N, Marchelidon J (1978) Effet d'hormones gonadotropes, in vitro, sur la concentration de l'adénosine monophosphate cyclique dans l'ovaire de l'anguille ( $A n-$ guilla anguilla L). Ann Biol Anim Biochim Biophys 18, 805-811
Leloup J, Brichon G, Hardy A (1976) Variations des hormones thyroïdiennes circulantes chez un Sélacien et des Téléostéens en fonction de l'évolution génitale et de la salinité du milieu. J Physiol, Paris 72, 48 A

Leloup-Hatey J, Hardy A, Quérat B (1989) Responses of gonadosomatic indice (GSI) and plasma levels of sex steroids to gonadotrophic (GTH) stimulation in freshwater European eel at yellow and silver stages. Gen Comp Endocrinol 74, 251

de Luze A, Leloup J (1984) Fish growth hormone enhances peripheral conversion of thyroxine to triodothyroxine in the eel (Anguilla anguilla L). Gen Comp Endocrinol 56, 308312

Le Menn F, Burzawa-Gérard E (1985) Effect of carp gonadotropin (CGTH) and a fraction unadsorbed on concanavalin A-Sepharose obtained from cGTH on vitelogenesis in the hypophysectomized marine teleost Gobius niger. Gen Comp Endocrinol 57, 23-36

Rodolico A (1933) Differenziamento dei sessi ed ovospermatogenese nell anguilla. Pubb/ Staz Zool Napoli 13, 180-278

Suzuki K, Kawauchi M, Nagahama Y (1988) Development of salmon GTH I and GTH II radioimmunoassays. Gen Comp Endocrinol 71, 450-458

Tesch FW (1979) The Eel. Biology and Management of Anguillid Eels. Chapman and Hall, London

Yamazaki $F$ (1965) Endocrinological studies on the reproduction of the female goldfish, $\mathrm{Ca}$ rassius auratus $\mathrm{L}$, with special reference to the function of the pituitary gland. Mém Fac Fish, Hokkaido Univ 13, 1-64 